\title{
A Probabilistic Approach to the Evaluation of Seismic Resilience in Road Asset Management
}

\author{
Vittorio Nicolosi ${ }^{1} \cdot$ Maria Augeri $^{1}$ - Mauro D'Apuzzo ${ }^{2}$ - Azzurra Evangelisti ${ }^{2}$. \\ Daniela Santilli ${ }^{2}$
}

Accepted: 13 January 2022/Published online: 15 February 2022

(C) The Author(s) 2022

\begin{abstract}
Road networks are classified as critical infrastructure systems. Their loss of functionality not only hinders residential and commercial activities, but also compromises evacuation and rescue after disasters. Dealing with risks to key strategic objectives is not new to asset management, and risk management is considered one of the core elements of asset management. Risk analysis has recently focused on understanding and designing strategies for resilience, especially in the case of seismic events that present a significant hazard to highway transportation networks. Following a review of risk and resilience concepts and metrics, an innovative methodology to stochastically assess the economic resources needed to restore damaged infrastructures, one that is a relevant and complementary element within a wider resilience-based framework, is proposed. The original methodology is based on collecting and analyzing ex post reconstruction and hazard data and was calibrated on data measured during the earthquake that struck central Italy in 2016 and collected in the following recovery phase. Although further improvements are needed, the proposed approach can be used effectively by road managers to provide useful information in developing seismic retrofitting plans.
\end{abstract}

Keywords Italy · Resilience metrics - Road asset management $\cdot$ Road network resilience $\cdot$ Seismic hazards

Vittorio Nicolosi

nicolosi@uniroma2.it

1 Department of Enterprise Engineering "Mario Lucertini", University of Rome "Tor Vergata", 00133 Rome, Italy

2 Department of Civil and Mechanical Engineering, University of Cassino and Southern Lazio, 03043 Cassino, Italy

\section{Introduction}

The launch of the 55000 series ISO Standards for Asset Management between 2014 and 2019 represented a step change for the asset management community. The standard series focus on managing physical and intangible assets and provide a framework to establish asset management policies, objectives, processes, and governance, and facilitate an organization's achievement of its strategic goals. The ISO standards point out that the risks and opportunities in managing assets need to be determined to prevent or reduce undesired effects (ISO 55001 2014; ISO 55002 2018).

With respect to risk assessment and management, reference is made to ISO 31000 standard launched in 2009 and revised in 2018. According to ISO 31000 (2018), risk is the effect of uncertainty on an organization's ability to meet its objectives, and risk management is a group of coordinated activities to direct and control an organization with regard to risk. The traditional definition of risk simply combines the probability of an event with its potential severity. Both definitions talk about the same phenomena but from two different perspectives. ISO thinks of risk in goal-oriented terms while the traditional definition thinks of risk in event-oriented terms.

Over the last two decades, risk management has shifted from risk reduction and protection of critical infrastructure assets from potential hazards, initially highly concentrated on direct threats, to improving infrastructure resilience (that is, the ability to absorb and quickly recover from events) to a wider range of hazards, including both direct and natural threats (Fekete et al. 2014).

The services provided by transportation and telecommunications systems, as well as water and electricity supply networks, are essential to our daily lives. The 
complexity of these networked systems has been increasing in recent decades, and has made them vulnerable. Transportation systems are among the infrastructure systems most affected by natural hazards and disasters (Keller and Atzl 2014; Utasse et al. 2016). Natural hazards are the main causes of transportation network disruptions in many countries, including Italy. Among these events, earthquakes are the costliest. Some good examples are the earthquakes that occurred in Sichuan, China (2008); Maule, Chile (2010); Tōhoku, Japan (2011); Christchurch, New Zealand (2011); and L'Aquila (2009) and Emilia (2012) in Italy. Therefore, incorporating risk management and resilience assessment into transportation asset management (TAM) procedures is an extremely important factor in order to manage events and not passively suffer the consequences of natural hazards and disasters, and an in-depth focus on this topic is needed.

\section{Resilience Definition and Metrics}

The traditional way of coping with adverse events is to develop approaches and systems to identify and mitigate risks. Stochastic models are used to forecast future adverse events and to make better-informed decisions about how to manage risk. These traditional risk management practices have proven insufficient to adequately address the adverse events that have occurred. Therefore, standards, legislations, and policies that are implemented to support protection needs of critical infrastructure have shifted their attention from identifying risk and alleviating the level of vulnerability to trying to increase resilience (Flannery et al. 2018).

Following the Hyogo Framework for Action 2005-2015 by the United Nations, definitions of resilience in transportation infrastructure systems have been discussed in some articles (Zhou et al. 2019). According to the literature, all definitions characterize resilience from one or both of two perspectives: (1) the capacity to maintain the serviceability (during a disaster event); and (2) the timing and resources required for restoring previous conditions (after a disaster event). Resilience of transport systems may be defined as the ability of transport systems to prepare for and adapt to a major disruption, providing and maintaining an acceptable level of service or functionality, and responding to and recovering rapidly from the disruption. Therefore, resilience measurement includes the time and level of performance the asset is able to sustain after an event occurs and before full performance is recovered (Fig. 1). The definition and illustration provided in Fig. 1 are consistent with approaches proposed worldwide, according to which resilience can be represented by four properties: robustness, redundancy, resourcefulness, and recovery speed. During the disruption phase, the levels of robustness and redundancy affect the performance level of a transportation system. Robustness, in particular, determines the capacity to resist damages caused by disaster events, and redundancy suggests the presence of alternative resources. During the recovery phase, both resourcefulness and rapidity indicate the ability of the system to recover serviceability.

In order to incorporate resilience into asset management processes, transport organizations need quantitative methods to measure the resilience of their systems to threats that may cause failures. Generally, there are two steps to measure a quantity. The first step consists of providing a metric for measurement, and the second step consists of computing the metric using some evaluation approaches. Currently, there is no unified measurement standard for resilience within road networks, both in terms of metrics and methods of assessment.

The metrics used so far for the resilience measurement of transportation infrastructure can be divided into two categories: topological metrics and performance-based metrics. Topological metrics look only at the network configuration of the transport system but ignore the operating conditions and usually use some topological characteristics of transportation networks, such as shortest path length (Schintler et al. 2007; Berche et al. 2009), average node degree (Testa et al. 2015), or betweenness centrality (Aydin et al. 2018). Performance-based metrics evaluate the resilience of the network systems based on their performance during all periods of time affected by disasters (disruption, time at bottom level, and recovery).

The three most widely used performance-based measures of resilience (MoR) are identified in the literature as: (1) the degradation of system quality over time; (2) the time-dependent function of quality during the restoring from disruption; and (3) the expected fraction of demand satisfied in the post-disaster network using specific recovery costs. Examples of performance-based MoR of type 1 are those proposed by Twumasi-Boakye and Frangopol (Bocchini and Frangopol 2012; Twumasi-Boakye and Sobanjo 2018) and by Adjetey-Bahun et al. (2016), and are represented by Eqs. 1 and 2, respectively:

$$
\begin{aligned}
& R=\int_{t_{0}}^{t_{3}}[100-Q(t)] \cdot d t \\
& R=\int_{t_{0}}^{t_{3}} \frac{[100-Q(t)]}{t} \cdot d t
\end{aligned}
$$

where $R$ is the value of resilience; $t_{0}$ is the time when the disruptive event occurs; $t_{3}$ is the time when the performance level of the system achieves the previous conditions 
Fig. 1 Phases of resilience measurement in road network functionality. PL $=$ Performance level

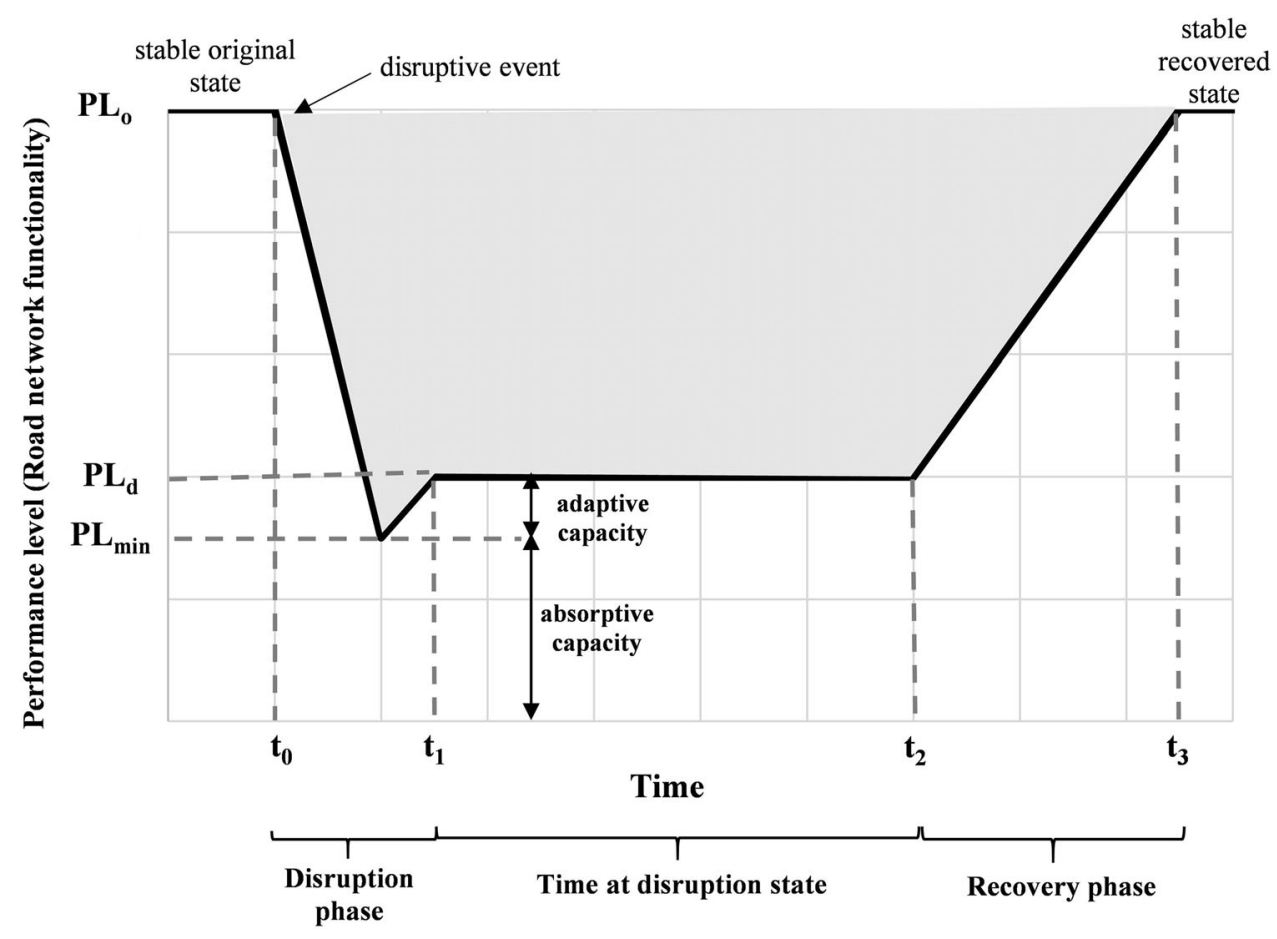

(see Fig. 1); and $Q(t)$ is the quality/performance of the system (for example, based on total travel time or total travel distance).

In type $2 \mathrm{MoR}$, resilience is dynamically represented as a temporal function, as opposed to the other types of indicators where resilience is a static indicator that represents the overall effects of disruption in the three phases following the occurrence of the event (see Fig. 1). An example of the performance-based MoR of type 2 is the one proposed by Liao et al. (2018) represented in Eq. 3:

$R\left(t_{r} \mid e_{j}\right)=\frac{F\left(t_{r} \mid e_{j}\right)-F\left(t_{d} \mid e_{j}\right)}{F\left(t_{0}\right)-F\left(t_{d} \mid e_{j}\right)}$

where $R\left(t_{r} \mid e_{j}\right)$ is the resilience value of the system at time $t_{r}$ resulting from disruption $e_{j} ; F\left(t_{r} \mid e_{j}\right)$ is the performance function of the system at time $t_{r}$ resulting from disruption $e_{j} ; F\left(t_{d} \mid e_{j}\right)$ is the minimum performance function of the system resulting from disruption $e_{j}$; and $F\left(t_{0}\right)$ is the function of the system at the pre-disruption state.

In type $3 \mathrm{MoR}$, resilience is defined as the expected fraction of demand satisfied by the network in the postdisaster phases with specific recovery costs. These indicators thus have two characteristics: (1) they explicitly consider transportation demand; and (2) they consider the dependence of the post-disruption phases on the economic resources committed. An example of metrics of type 3 is provided by Vugrin et al. (2014), who proposed to assess resilience through the system of relationships shown in Eq. 4:
$R=I S P+R C$

where ISP is the increase in cost of transport, including both increases in costs for flows on links and penalty costs for demand that cannot be accommodated; and $R C$ is the resources needed to implement the scheduled tasks on damaged links in the network. If resilience is to be measured according to this performance-based MoR approach, road managers should aim at minimizing the $R$ value pertaining to their transport network.

In general, many researchers consider the performancebased metrics more suitable than topological metrics to measure the resilience in transportation networks, as the latter do not consider directly the effect on the users. Among the performance-based metrics, type 3 is often preferred over the other two because it takes into account the performance offered by the system during the entire time frame in which it was affected/disturbed by the disruptive event. Furthermore, the metrics that incorporate the economic resources required for recovery, which influence the recovery time, into the resilience evaluation, are better than the others, which usually fix as constraints the recovery resources.

In the literature numerous approaches have been proposed to measure the level of service of transportation systems and calculate performance-based resilience metrics. These performance evaluation approaches are classified as: optimization models; simulation models; probability theory models; fuzzy logic models; and datadriven models. Since the purpose of this study is not a 
literature review, the different approaches are not analyzed in detail, but optimization approaches are the most widely used (Zhou et al. 2019). They are primarily used to address two types of problems: (1) solving the traffic assignment problem, through deterministic or stochastic user equilibrium (UE) assignment algorithms; and (2) optimizing the utility of resources deployed for mitigation/preparation/ response/recovery.

In this study, a metrics for evaluating the seismic resilience is proposed, based on both cumulative impact of disruption on system performance and the recovery efforts/costs. An operational procedure for stochastic evaluation of roadway rehabilitation costs is illustrated. The procedure was calibrated using data acquired for road infrastructure restoration during the 2016 earthquake in central Italy.

\section{Seismic Resilience Evaluation Methodology}

In this section the proposed procedure to stochastically evaluate rehabilitation costs due to a seismic event is presented. The discussion on the proposed methodology is first framed in a wider context pertaining to the resiliencebased performance evaluation for transportation networks. Subsequently, following the stochastically-based framework, the issue related to the assessment of recovery cost is addressed by highlighting the contributions by the seismic hazard and by the vulnerability of critical transportation assets.

\subsection{The Metrics for Assessing Seismic Resilience}

The objective is to introduce a methodology for the quantitative and stochastic assessment of the resilience of road networks to earthquake hazards. As resilience evaluations have to be considered as part of risk management in transportation asset management (TAM), the proposed methodology is consistent with the risk analysis criteria introduced in the ISO 31000 (2018) standards. In addition, the approach used to describe the randomness of seismic events complies with the procedures developed within the European design standards (that is, EN 1998-1 2004).

The methodology developed in this study refers to a performance-based measure and a metric of the third type that explicitly considers the resources required for recovery, as explained in Sect. 2. Specifically, a cost-based resilience indicator composed of two addendums-the increase in the cost of transportation on the road network, and the cost of restoring the infrastructure following the occurrence of the seismic event-is proposed that can be expressed by means of the following:

$$
\left\{\begin{array}{l}
R_{r}=O D C_{r}+O R C_{r} \\
O D C_{r}=\sum_{i}\left[G T C_{i \_p o s t}\left(R C_{i, r}\right)-G T C_{i \_p r e}\right] \\
O R C_{r}=\sum_{i} \sum_{j} R C_{i, j}\left(r, V_{i, j}\right)
\end{array}\right.
$$

where $O D C_{r}$ is the overall delay cost of transport on the road network caused by an earthquake at a reliability level $r$ (which has $r$ probability of not being exceeded during the period of analysis); $G T C_{i_{\text {ppost }}}\left(R C_{i, r}\right)$ is the generalized transport cost on the link $i$ in the post-earthquake scenario that has $r$ probability of not being exceeded during the period of analysis; $G T C_{i_{-} p r e}$ is the generalized transport cost on the link $i$ in the pre-earthquake scenario; $O R C_{r}$ is the overall recovery cost at a reliability level $r ; R C_{i, j}\left(r, V_{j, j}\right)$ is the recovery cost function on the element $j$ (for example, bridge, tunnel,and so on) of the link $i$ (having as independent variable the vulnerability $V$ of the element and the reliability $r$ ).

The cost of transport on the different links of the network is determined by redistributing traffic on the road network after and before the earthquake by solving the traffic assignment problem, through user equilibrium assignment algorithms. These issues and the evaluation of the $O D C_{r}$ component have been discussed by D'Apuzzo et al. (D'Apuzzo, Esposito, et al. 2019; D'Apuzzo, Evangelisti, et al. 2019; D'Apuzzo et al. 2020). This study focused on a methodology developed for the evaluation of the recovery cost, and in particular the recovery cost functions calibrated on the basis of data acquired during the 2016 earthquakes in central Italy.

\subsection{Stochastic Recovery Cost Function for the Resilience Evaluation}

In the method proposed in this study for risk management, the effects are measured in terms of economic damage $D$, or rather resources needed to restore infrastructure functionality after an event. The effects or consequences of a natural event in general, and an earthquake in particular, depend on two factors:

- Magnitude of the action that for earthquakes is measured by the PGA parameter (that is, maximum or peak ground acceleration that may occur during an earthquake in a specific location);

- Vulnerability of the infrastructure, that is, the ability of the construction to withstand actions of a certain type.

Provided the stochastic nature of the involved factors, the probability that during a period of time $T$, an economic damage higher than or equal to $D$ occurs in an asset element having vulnerability $V$, can be expressed by the following: 


$$
F(D, T, V)=P(D, V \mid T, P G A) \cdot P(T, P G A)
$$

where $F(D, T, V)$ is the probability that, during a period of time $T$, an economic damage higher than or equal to $D$ occurs in an asset element having vulnerability $V ; P(D, V$ I $T, P G A$ ) is the conditional probability of having a damage greater than or equal to $D$ in an asset element having vulnerability $V$ for a given earthquake with a magnitude higher than or equal to $P G A$ in a period of time $T ; P(T$, $P G A$ ) is the probabilistic seismic hazard function (that is, probability of having almost one earthquake with a magnitude higher than or equal to $P G A$ in a period of time T) defined below.

\subsubsection{Seismic Risk Assessment}

Seismic hazard maps that provide peak ground acceleration $(P G A)$ at all sites for different probability of exceedance $(P E)$ and/or for different return periods $T_{R}$, are currently used in many European countries. The inverse of the return period is the average annual frequency recurrence $\lambda$ of the event linked to that intensity:

$\lambda=\frac{1}{T_{R}}$

The plot of the values allows us to obtain the hazard curve.

Normally the occurrence of a ground motion parameter at a site in excess of a specified level is modelled as Poisson process. Clearly this implies that any seismic event is independent of the occurrence of all others, and this could be approximately true for major earthquakes, excluding associated foreshocks, aftershocks, and so on. Applying this stochastic model, the probability of occurrence of $n$ events, which have intensity (PGA) higher than the one corresponding to the average frequency of recurrence $\lambda$, in a time interval $t$, is:

$P(n)=P_{n}=\frac{(\lambda \cdot t)^{n}}{n !} \cdot \mathrm{e}^{-\lambda \cdot t}$

Consequently, it is possible to evaluate the recurrence law to predict the probability of at least one exceedance in a period $t$ :

$P(n \geq 1)=1-P_{n=0}=1-\mathrm{e}^{-\lambda \cdot t}$

An example of a probabilistic hazard curve is shown in Fig. 2.

\subsubsection{Cost Damage Function}

Although the probabilistic seismic hazard function can be easily evaluated by means of a seismic hazard curve, evaluation of the conditional probability $P(D, V \mid T, P G A)$ of

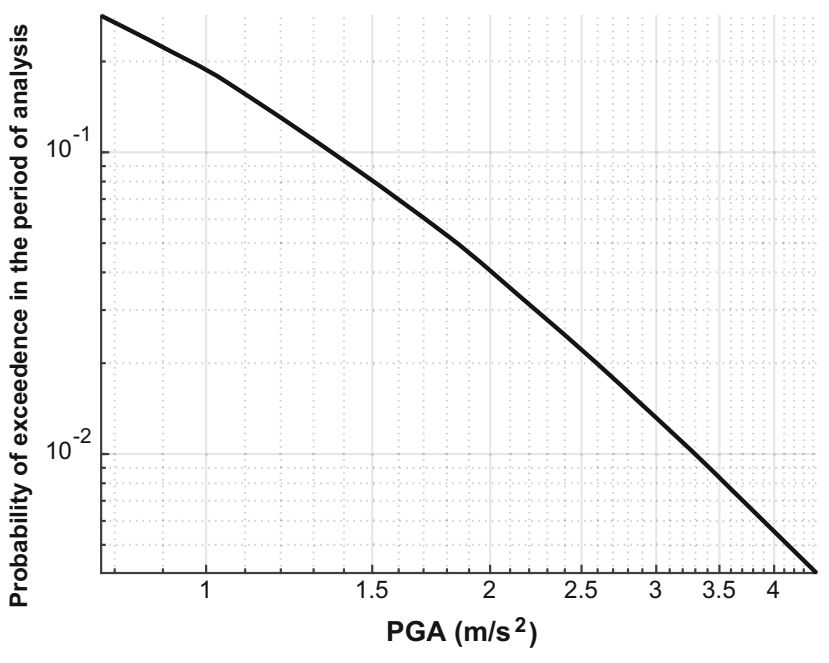

Fig. 2 Example of a typical hazard curve in a site for a time period of 10 years. PGA $=$ Peak ground acceleration

having a damage greater than or equal to $D$ in an asset element having vulnerability $V$ for a given earthquake with a magnitude higher than or equal to $P G A$ in a period of time $T$ is more complex, since it requires information on: the vulnerability of different road sections; the damage the different road assets underwent following a real seismic event; the corresponding seismic magnitude of the analyzed event (also evaluated according to site conditions); and the corresponding recovery or reconstruction cost borne to restore the ex ante serviceability conditions.

Since all these data are of stochastic nature, an economic damage function, $D$, can be represented through a stochastic function:

$D=f(P G A, V)$

where $f(P G A, V)$ is the cost damage function that returns the average economic damage in an element of the transportation asset having vulnerability $V$ when an earthquake of "PGA" magnitude occurs.

Therefore if data on recorded $P G A$ and on vulnerability values for the different road assets (viaduct, tunnel, embankment, trenches) can be collected for a wide area subjected to a given seismic event, it is possible to empirically derive a functional form for the economic damage function, $D$, by combining all the collected information.

\subsubsection{Vulnerability}

The seismic vulnerability of a structure is a quantity associated with its weakness/strength in the case of earthquakes of a given intensity, and expresses the probability that buildings will suffer damage due to a seismic phenomenon. Different seismic vulnerability assessment 
methods have been proposed in the literature and can be divided into three main groups-empirical methods, analytical methods, and hybrid methods (Shabani et al. 2021).

Many of the analytical methods share the same assumption that the gap (or ratio) between the actual seismic demand and the seismic capacity of the structure (or the seismic demand at the time of the design) can be considered as an indicator of structural performance deficit. Therefore, in this research, the following quantitative seismic structural performance parameter is used to assess vulnerability:

$R_{P G A i}=\frac{P G A_{C i}}{P G A_{D i}}$

where $i$ is the limit state considered (for example, ultimate limit state or serviceability limit state); $R_{P G A i}$ is the structural performance indicator in terms of $P G A$ for limit state $i$; $P G A_{C i}$ is the maximum extent of the actions that the structure is able to support, for limit state $i$, in accordance with the margins of safety imposed by the standards (that is, peak ground acceleration "capacity"); $P G A_{D i}$ is the expected seismic action stated by the standard for limit state $i$ (that is, peak ground acceleration "demand").

Values of the $R_{P G A i}$ indicator are typically between 0 and 1: values close to 1 characterize cases where the reliability level is close to the one required by the standards; values close to 0 represent a much lower reliability than that allowed by the standards (high risk cases). The $R_{P G A i}$ indicator is analogous to the structural performance score (SPS) included in the New Zealand guidelines for assessing and improving the seismic performance of buildings during earthquakes (NZSEE 2014), or to the NODE indicator proposed by Iervolino (Petruzzelli and Iervolino 2021), and the quantity $1-R_{P G A i}$ is related to the probability of damage (that is, vulnerability). Therefore, the $R_{P G A i}$ indicator has been used in this study to evaluate vulnerability (Table 1). Once all the involved factors had been defined, the proposed methodology was applied to data collected following a major seismic event that struck central Italy in 2016. Collected data and corresponding derived damage cost functions are reported below.

Table 1 Vulnerability classes adopted by the current study for bridges (ultimate limit state, ULS)

\begin{tabular}{ll}
\hline$R_{P G A}$ & Vulnerability class \\
\hline$R \geq 0.8$ & A (low) \\
$0.4 \leq R<0.8$ & B (medium) \\
$\mathrm{R}<0.4$ & C (high) \\
\hline
\end{tabular}

\section{Data}

In this study, using the indicators previously illustrated, three classes of vulnerability were introduced, based on the $R_{P G A}$ (Table 1). In order to develop a recovery cost function, the data on the costs of the interventions on the national road network and PGAs recorded in the seismic events in central Italy during the years 2016-2017 were used. The analysis of the costs for the renewal of the road network was carried out on a regional scale, as extensive damage affected nine provinces (Fig. 3): Ascoli Piceno, Rieti, Macerata, Fermo, Perugia, Teramo, L'Aquila, Ancona, and Terni. The intensity of the seismic events that affected the territory in question in 2016 is reflected by the epicenters:

- 24 Aug 2016, Epicenter at Accumoli (Rieti), Moment Magnitude Scale (MMS) 6,0;

- 26 Oct 2016, Epicenter at Visso (Macerata), MMS 5,9;

- 30 Oct 2016, Epicenter at Norcia (Perugia), MMS 6,5.

Recovery and repair interventions were foreseen for several damaged engineering works, which were divided into two macro-groups: road sections (excavation/embankment) and bridges. The first one includes: road pavements, retaining walls, bulkheads, and every typology of complementary works designed to protect and support the road. The second one includes all types of bridges, regardless of construction technology. For each of the provinces affected by the earthquakes, the total costs related to the interventions on the roads and bridges were assessed. The former was related to the length of the road network (excluding bridges), the latter to the total length of the bridges existing in the road network; therefore, it was possible to obtain costs per unit length. These values are listed in Table 2 (last two columns).

To characterize the intensity of the seismic events, the PGA was selected as the reference seismic parameter. Since the aim was to perform an analysis with a regional extension, the effects of local amplification of ground motion were not considered. Therefore, the data of the ground accelerations recorded during the seismic events were processed. To perform this operation, the database of Project Orfeus, called the Engineering Strong-Motion Database, was used (Luzi et al. 2016). It allowed us to obtain all the engineering seismic parameters recorded by acceleration stations during the ground shaking, such as ground acceleration, velocity, displacement, and response spectrums.

For each of the seismic events, the data relative to the peak ground accelerations recorded by 60 accelerometric stations in the area of interest were analyzed. Subsequently, a mean value, restricted to administrative boundaries and 


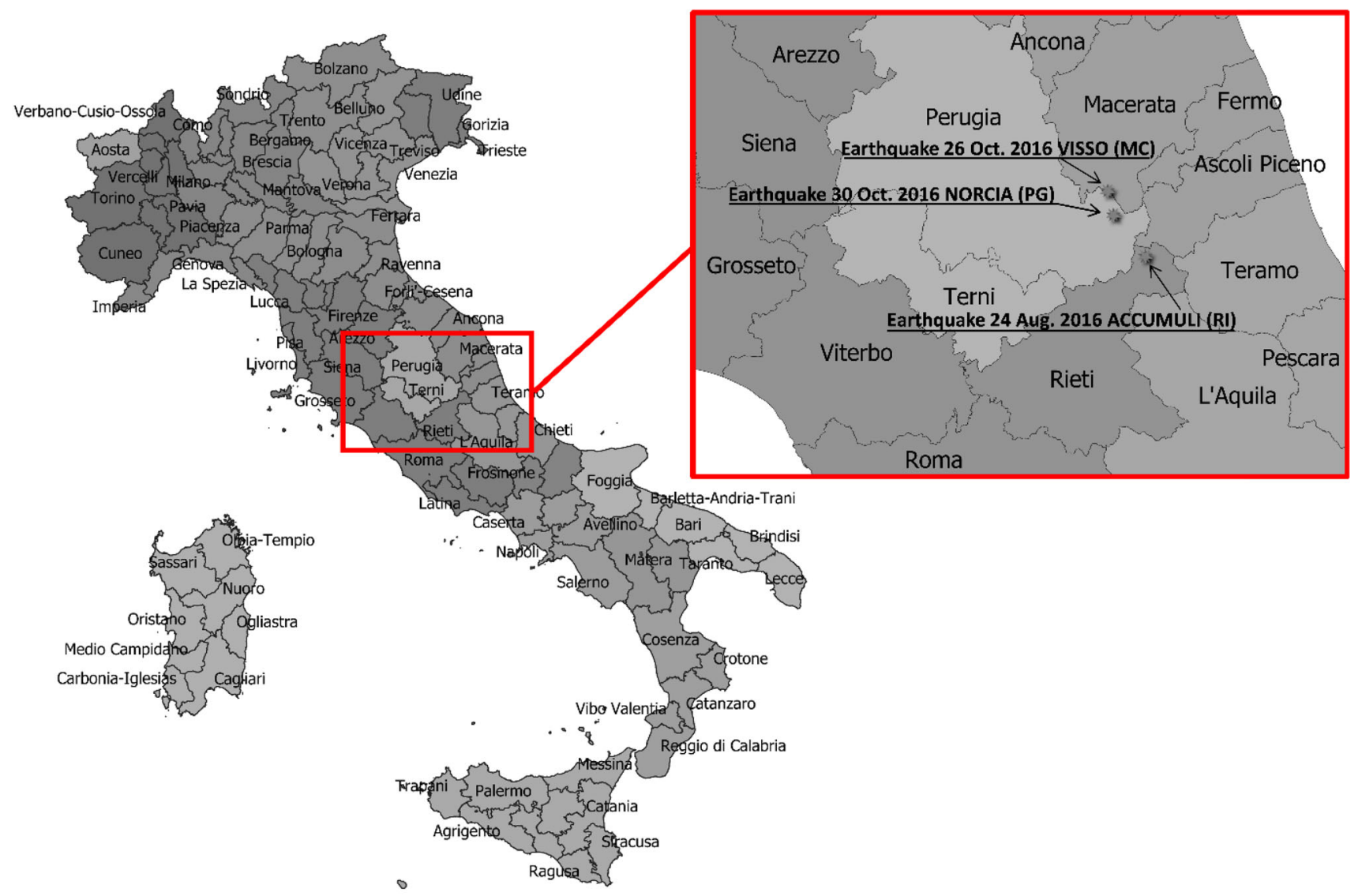

Fig. 3 Map of Italy showing the study area and the affected provinces and epicenters of the earthquakes considered

Table 2 Repair costs per unit length of roads and bridges in the central Italian study area road network and average values of peak ground accelerations (PGAs) recorded during the earthquakes, compared with the values provided for the ultimate limit state (ULS) by the construction standards (NTC 2018)

\begin{tabular}{lllcl}
\hline Provinces & Regional PGA $\left(\mathrm{m} / \mathrm{s}^{2}\right)$ & PGA $(\mathrm{ULS})\left(\mathrm{m} / \mathrm{s}^{2}\right)$ & Road body repair cost $(\mathrm{k}$ USD $/ \mathrm{km})$ & Bridges repair cost $(\mathrm{k}$ USD/km) \\
\hline Ascoli Piceno & 3.09 & 3.08 & 193.6 & 8075.5 \\
Rieti & 2.47 & 2.95 & 49.3 & 5353.5 \\
Macerata & 2.43 & 3.12 & 100.6 & 1227.6 \\
Fermo & 1.46 & 3.31 & 27.2 & 150.5 \\
Perugia & 1.40 & 3.03 & 23.0 & 2003.1 \\
Teramo & 1.01 & 3.12 & 6.7 & 307.3 \\
L'Aquila & 0.86 & 4.44 & 0.5 & - \\
Ancona & 0.64 & 3.12 & 3.0 & 45.0 \\
Terni & 0.32 & 2.64 & 0.4 &
\end{tabular}

damaged areas, was calculated (see the first column of Table 2).

Additional data related to the ultimate limit state of collapse (ULS) can be added to the data collected and previously illustrated. The main European and US regulations promote the principle according to which the "nocollapse" requirement (that is, "The structure shall be designed and constructed to withstand the design seismic action without local or global collapse, thus retaining its structural integrity and a residual load bearing capacity after the seismic events" (EN 1998-1 2004, paragraph 2.1, p. 29)) is achieved for design seismic action associated with a low reference probability of exceedance $(5-10 \%)$ in a reference period (for example, 50-200 years) (EN 
1998-1 2004; AASHTO 2010; FHWA 2014; NTC 2018). In this case study of central Italy, the following criterion was chosen: $5 \%$ reference probability of exceedance in a reference period of 200 years (class IV suggested for national two-lane highway and motorway according to NTC 2018). The choice is based on both the necessary agreement between the experimental data and related Italian regulations, and above all, the most precautionary target. However, the criteria and thresholds chosen are consistent with those adopted in most of the more economically developed countries. Therefore, the seismic action linked to this return period can be associated with the total reconstruction cost of the road element. On the basis of analyses carried out by the Ministry of Transport, the parametric cost of reconstruction (carriageway about 12 $\mathrm{m}$ wide) for the bridges and the road sections (general road cross-section excavation or embankment) is $12,680.40$ and $2,488.00$ thousand $\mathrm{USD} / \mathrm{km}$, respectively.

Finally, it must be considered that the cost for the same seismic action depends on the vulnerability. Vulnerability data were provided by the national road organization ANAS (Azienda Nazionale Autonoma delle Strade/Italian National Road Company). This allowed the identification of low and high vulnerability infrastructural systems, with reference to the ultimate limit state (ULS) risk values of Table 1 . For ordinary road sections there is currently no classification according to vulnerability so it has not been possible to distinguish the data.

\section{Results}

The data on earthquake intensity and road damage collected allowed the development of cost functions through statistical regressions. Considering that these functions must tend to zero for PGA $=0$ and to cost of reconstructions for PGA of the ultimate limit states, it was decided to use a sigmoidal function. The cost functions identified are shown below and represented for bridges and excavation/ embankment road sections, respectively, in Figs. 4 and 5:

$D=a+\frac{b}{(1+\exp (\mathrm{c} \cdot P G A+\mathrm{d}))}$

where $a=-\frac{b}{(1+\exp (d))}, \quad b$ is the maximum cost corresponding to the cost of reconstruction (Table 3); $\mathrm{c}$ is a constant that governs the form of the function and is assumed to be the same for all levels of vulnerability (Table 3); and d is a constant, or rather a translation factor, which depends on the level of vulnerability (Table 3).

Because there are no vulnerability data for ordinary road sections, the regression function obtained has been attributed to a medium vulnerability, and the functions for high and low vulnerability have been obtained by shifting this function similarly to that found for bridges.

Due to their generality, cost functions can be considered valid for the whole national territory. Finally, the introduced functions represent two elements of seismic risk analysis: vulnerability (V), and exposure (E). The cost functions identified so far are stochastic and link the PGA to the damages. To account for the random effects of the seismic phenomenon, it is necessary to use the stochastic model shown earlier for PGA (see Sect. 3.2.1 and Eq. 8).

After defining a period of analysis (for example, $T=10$ years, see Fig. 2) the hazard curves can be obtained. Using the cost function determined earlier, it is possible to substitute the recovery cost for the PGA in the hazard curve. This yields a stochastic representation of the recovery cost due to seismic events $R C i, j\left(r, V_{i, j}\right)$ (Fig. 6), which is one of the addendums of the resilience indicator to be used in road asset management, as mentioned in Sect. 3.1. With the reliability (that is, the probability to not exceed a cost) decided and the vulnerability of the element known, the expected recovery cost is obtained. This result can also be useful in the evaluation of budget plans for future retrofitting interventions by highway network managers, and since the employed vulnerability and hazard criteria are consistent with the regulations adopted in the European Union and the United States, they may represent an effective tool for most highway managers worldwide.

Moreover, although it is fair to assume that design and construction techniques for bridges and excavation/embankments road sections are similar in most of the more economically developed countries, the sigmoidal function allows to "scale" reconstruction costs (by choosing a suitable value for the $b$ parameter) conveniently in order to adapt the evaluation to the specific national economic situation.

\section{Conclusion}

Introducing resilience into road asset management systems requires the establishment of effective metrics and robust systems for evaluating them. This article presents a stochastic metric for assessing the resilience of road networks against disruptive seismic events and a framework for performing its evaluation. Given that the ultimate goal of a road network's resilience is to ensure the continuity of its normal functions, the metric chosen is based on changes in total costs (that is, maintenance/restoration costs and transportation costs). The cost-based assessment allows for the quantification of resilience, for all three phases usually considered (see Fig. 1), using a single unit of measure for the different addendums. 
Fig. 4 Empirical cost function with three vulnerability levels for bridges in the central Italian study area

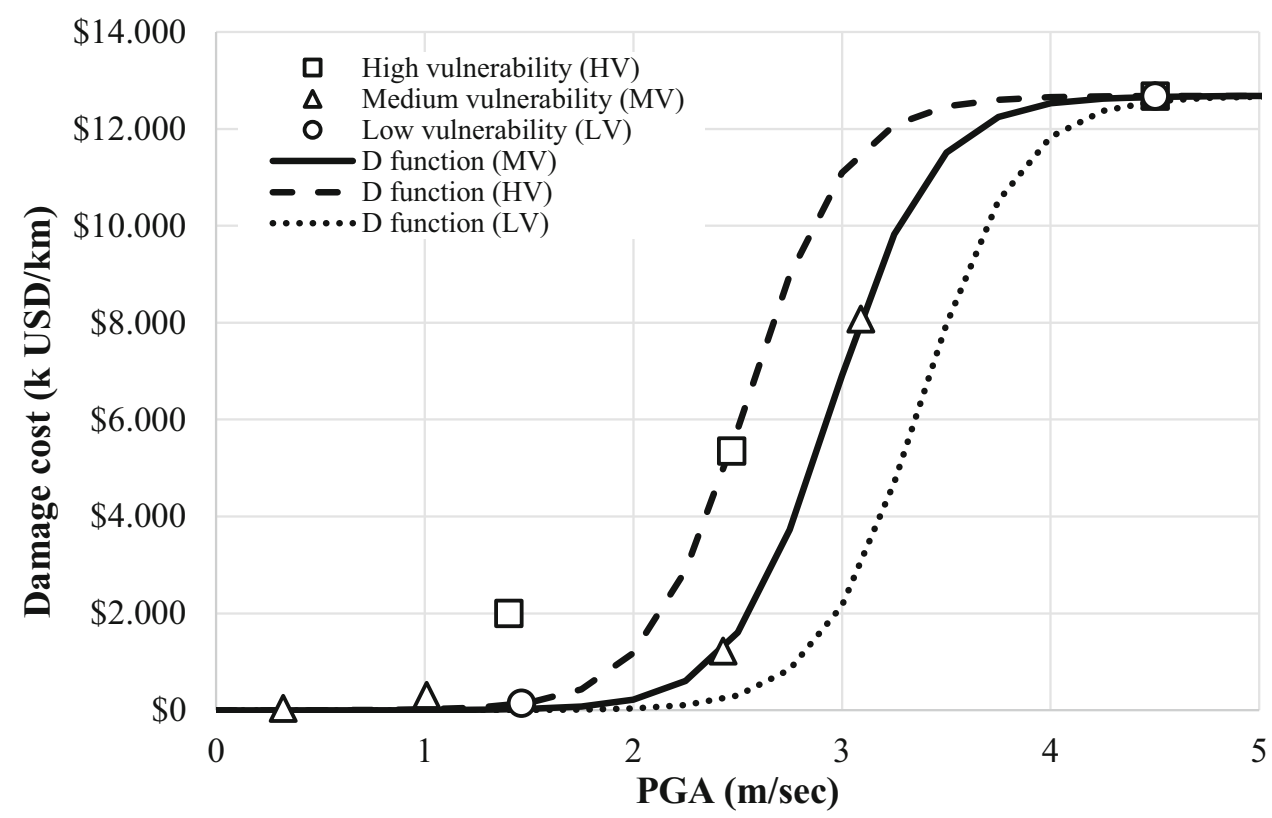

Fig. 5 Empirical cost function with three vulnerability levels for roads in the central Italian study area

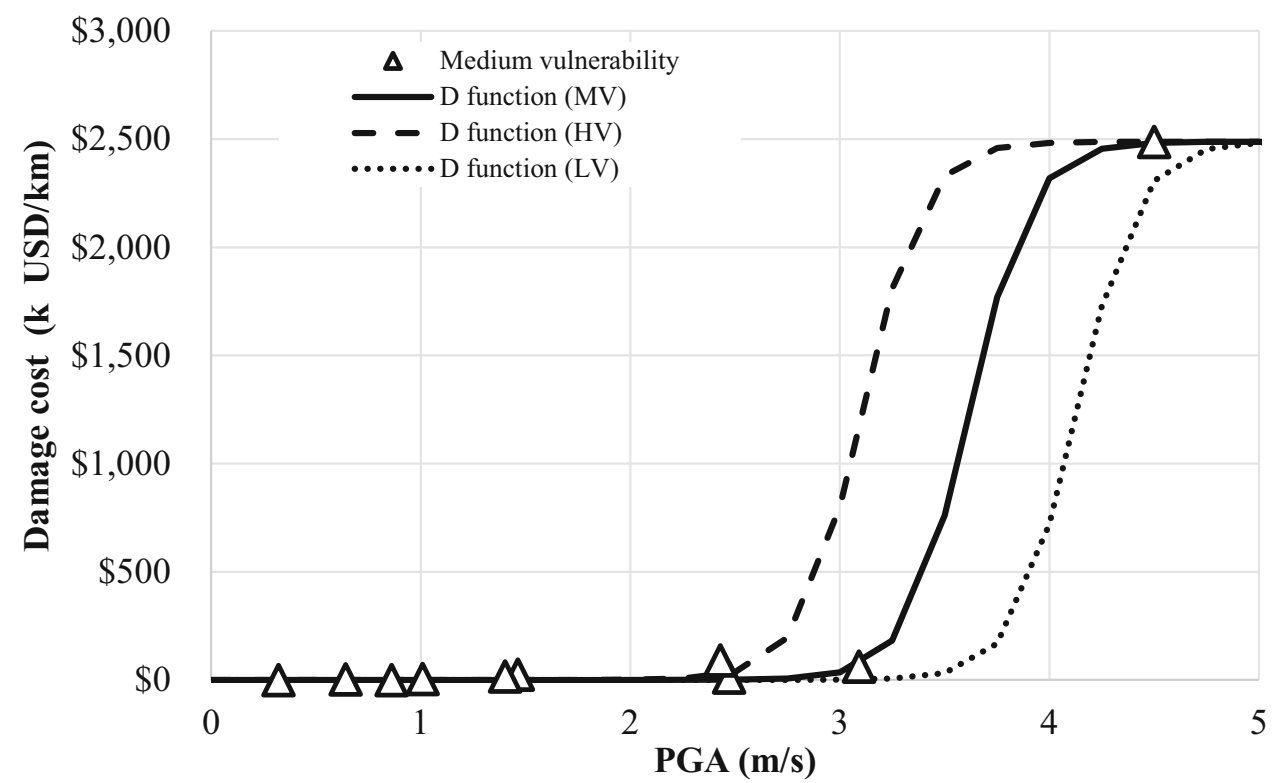

Table 3 Parameters of the sigmoidal cost function for assets, bridges, and road sections related to the three levels of vulnerability in the central Italian study area

\begin{tabular}{|c|c|c|c|c|c|c|}
\hline \multirow[t]{2}{*}{ Function Parameters } & \multicolumn{3}{|l|}{$\begin{array}{l}\text { Bridge } \\
\text { vulnerability }\end{array}$} & \multicolumn{3}{|c|}{ Excavation/embankment road sections vulnerability } \\
\hline & Medium & High & Low & Medium & High & Low \\
\hline $\begin{array}{l}\mathrm{b} \\
(\mathrm{k} \text { USD/km) }\end{array}$ & $\$ 12,680.40$ & $\$ 12,680.40$ & $\$ 12,680.40$ & $\$ 2488.00$ & $\$ 2488.00$ & $\$ 2488.00$ \\
\hline $\mathrm{c}$ & -4.2219 & -4.2219 & -4.2219 & 6.8888 & 6.8888 & 6.8888 \\
\hline d & 12.4867 & 10.7228 & 14.2507 & 24.9330 & 21.4110 & 28.4551 \\
\hline
\end{tabular}




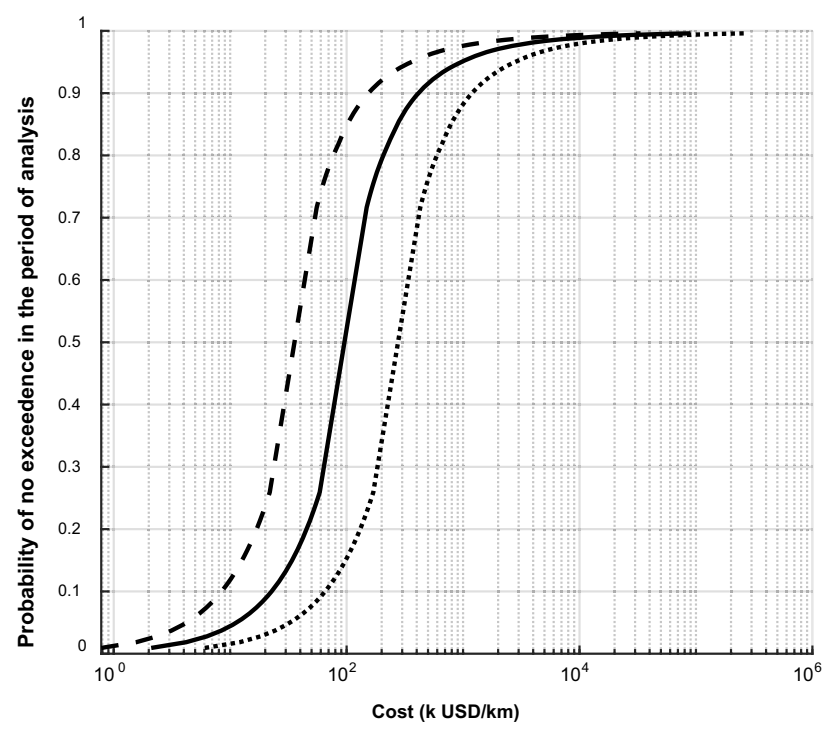

Fig. 6 Probability of not exceeding the repair cost in the period of analysis for bridges (solid line: medium vulnerability; dotted line: high vulnerability; dashed line: low vulnerability)

In the study, attention was focused on the assessment of damage/repair costs of the road elements (embankments, cuttings, bridges, and viaducts) damaged by seismic events (absorptive capacity). These costs, in addition to constituting one of the addendums of the resilience indicator, are a fundamental element for evaluating possible damage and reductions in the functionality of the various links of the road network and the consequent increases in the cost of transport.

The proposed criterion for evaluating repair cost after an earthquake is aligned with the design standards (see, for example EN 1998-1 2004) both in terms of defining seismic risk, that is, the probability of having a seismic event of a given magnitude (PGA), and in terms of defining the vulnerability of infrastructure elements. This feature makes it possible to establish a transparent and direct link between any planned and designed retrofitting interventions on infrastructure elements and the increase in their resilience characteristics.

The proposed criterion has been applied by referring to the data available on the national Italian road network during the earthquakes in central Italy (2016-2017). The case study shows how on the basis of historical data it is possible to perform a calibration of the stochastic evaluation tool of the costs of restoration of a road network. The assessment tool, once calibrated, allows the quantification of the resilience of the network related to the recovery phase for future seismic events as a function of maintenance interventions aimed at decreasing the vulnerability of the infrastructure. In the application to the case study, some critical issues emerged for the assessment of repair costs of cuttings and embankments of a road section, due to the almost complete absence of a vulnerability classification for these infrastructure elements.

Future research will be directed toward integrating the economic damage assessment criterion into the transportation cost assessment. In particular, on the basis of real data the possibility of establishing a correspondence between the repair costs required following an earthquake of a given intensity and the limitations to road traffic caused by the damage itself will be verified. This will allow the completion of the structure for the evaluation of the proposed resilience indicator.

However, since hazard and vulnerability criteria have been derived from international standards and regulations, the proposed methodology, but also the obtained damage cost functions themselves, may provide an effective and immediate evaluation tool for road managers worldwide as far as future budget scenarios for road infrastructure seismic retrofitting are concerned since the required input parameters on the specific examined road network can be easily collected.

Although it can be argued that the proposed stochastic damage/repair cost function has been developed and calibrated based only on Italian data, the design and construction techniques are quite similar for most of the more economically developed countries. However, the employed sigmoidal structure allows the adjustment of the evaluation tool to the specific country economic situation by choosing an appropriate value for the unit reconstruction cost.

Therefore, the framework of stochastic cost recovery functions developed may represent an interesting and sound basis for road managers who need to evaluate financial resources to be gained in order to implement the restoring operations following an earthquake. This is the case because the stochastic character of the presented approach, that allows the definition of a specific exceedance probability, can provide a sound justification for retrofitting budget needs as far as technical issues are concerned, provided that a convenient shift on repair and reconstruction costs is to be assessed based on the specific country economic situation.

Acknowledgements We thank the Operational and Territorial Coordination Directorate of the Italian National Road Company (ANAS) for the information provided on the interventions carried out on the national road network following the earthquake that hit central Italy in 2016. We would also like to thank engineer Claudio Petricca for his support in the collection of data and in the analysis operations.

Open Access This article is licensed under a Creative Commons Attribution 4.0 International License, which permits use, sharing, adaptation, distribution and reproduction in any medium or format, as long as you give appropriate credit to the original author(s) and the source, provide a link to the Creative Commons licence, and indicate if changes were made. The images or other third party material in this article are included in the article's Creative Commons licence, unless indicated otherwise in a credit line to the material. If material is not 
included in the article's Creative Commons licence and your intended use is not permitted by statutory regulation or exceeds the permitted use, you will need to obtain permission directly from the copyright holder. To view a copy of this licence, visit http://creativecommons. org/licenses/by/4.0/.

\section{References}

AASHTO (American Association of State Highway and Transportation Officials). 2010. LRFD bridge design specifications, 5th edn. Washington, DC: AASHTO.

Adjetey-Bahun, K., B. Birregah, E. Châtelet, and J.L. Planchet. 2016. A model to quantify the resilience of mass railway transportation systems. Reliability Engineering and System Safety 153: 1-14.

Aydin, N.Y., H.S. Duzgun, F. Wenzel, and H.R. Heinimann. 2018. Integration of stress testing with graph theory to assess the resilience of urban road networks under seismic hazards. Natural Hazards 91(1): 37-68.

Berche, B., C. von Ferber, T. Holovatch, and Y. Holovatch. 2009. Resilience of public transport networks against attacks. The European Physical Journal B 71(1): 125-137.

Bocchini, P., and D.M. Frangopol. 2012. Optimal resilience- and costbased postdisaster intervention prioritization for bridges along a highway segment. Journal of Bridge Engineering 17(1): $117-129$.

D’Apuzzo, M., A. Esposito, A. Evangelisti, R.-L. Spacagna, L. Paolella, and G. Modoni. 2019. Strategies for the assessment of risk induced by seismic liquefaction on road networks. In Proceedings of the 29th European Safety and Reliability Conference, ed. M. Beer, and E. Zio, 1651-1659. Singapore: Research Publishing.

D’Apuzzo, M., A. Evangelisti, G. Modoni, R.-L. Spacagna, L. Paolella, D. Santilli, and V. Nicolosi. 2020. Simplified approach for liquefaction risk assessment of transportation systems: Preliminary outcomes. In Computational science and its applications-ICCSA 2020, eds. O. Gervasi, B. Murgante, S. Misra, C. Garau, I. Blečić, D. Taniar, B.O. Apduhan, and A.M.A.C. Rocha et al., 130-145. Cham, Switzerland: Springer.

D'Apuzzo, M., A. Evangelisti, V. Nicolosi, A. Rasulo, D. Santilli, and M. Zullo. 2019. Simplified approach for the prioritization of bridge stock seismic retrofitting. In Proceedings of the 29th European Safety and Reliability Conference, ed. M. Beer, and E. Zio, 3277-3284. Singapore: Research Publishing.

EN 1998-1. 2004. Eurocode 8: Design of structures for earthquake resistance-Part 1: General rules, seismic actions and rules for buildings. Brussels: European Committee for Standardization.

Fekete, A., G. Hufschmidt, and S. Kruse. 2014. Benefits and challenges of resilience and vulnerability for disaster risk management. International Journal of Disaster Risk Science 5(1): 3-20.

FHWA (Federal Highway Administration). 2014. LRFD seismic analysis and design of bridges: Reference manual. FHWA-NHI15-004. Washington, DC: FHWA. https://www.fhwa.dot.gov/ bridge/seismic/nhi130093.pdf. Accessed 13 Jan 2022.

Flannery, A., M.A. Pena, and J. Manns. 2018. Resilience in transportation planning, engineering, management, policy, and administration. Washington, DC: The National Academies Press.
ISO 31000. 2018. Risk management-Guidelines. Technical committee: ISO/TC 262 risk management. Geneva, Switzerland: International Organization for Standardization (ISO).

ISO 55001. 2014. Asset management-Management systemsRequirements. Geneva, Switzerland: International Standards for Organisation (ISO).

ISO 55002. 2018. Asset management-Management systems-Guidelines for the application of ISO 55001. Geneva, Switzerland: International Standards for Organisation (ISO).

Keller, S., and A. Atzl. 2014. Mapping natural hazard impacts on road infrastructure - the extreme precipitation in Baden-Württemberg, Germany, June 2013. International Journal of Disaster Risk Science 5(3): 227-241.

Liao, T.-Y., T.-Y. Hu, and Y.-N. Ko. 2018. A resilience optimization model for transportation networks under disasters. Natural Hazards 93(1): 469-489.

Luzi, L., R. Puglia, E. Russo, M. D’Amico, C. Felicetta, F. Pacor, G. Lanzano, and U. Çeken et al. 2016. The engineering strongmotion database: A platform to access Pan-European accelerometric data. Seismological Research Letters 87(4): 987-997.

NTC (Norme tecniche per le costruzioni). 2018. Italian regulation. Decreto Ministeriale 17 Gennaio 2018. Aggiornamento delle "Norme tecniche per le costruzioni". G.U. n.42 del 20 Febbraio 2018 (in Italian).

NZSEE (New Zealand Society for Earthquake Engineering). 2014. Assessment and improvement of the structural performance of buildings in earthquakes: Prioritisation, initial evaluation, detailed assessment, improvement measures: Recommendations of a NZSEE study group on earthquake risk buildings. Wellington: New Zealand Society for Earthquake Engineering.

Petruzzelli, F., and I. Iervolino. 2021. NODE: A large-scale seismic risk prioritization tool for Italy based on nominal structural performance. Bulletin of Earthquake Engineering 19(7): 2763-2796.

Schintler, L.A., R. Kulkarni, S. Gorman, and R. Stough. 2007. Using raster-based GIS and graph theory to analyze complex networks. Networks and Spatial Economics 7(4): 301-313.

Shabani, A., M. Kioumarsi, and M. Zucconi. 2021. State of the art of simplified analytical methods for seismic vulnerability assessment of unreinforced masonry buildings. Engineering Structures 239: Article 112280.

Testa, A.C., M.N. Furtado, and A. Alipour. 2015. Resilience of coastal transportation networks faced with extreme climatic events. Transportation Research Record 2532(1): 29-36.

Twumasi-Boakye, R., and J.O. Sobanjo. 2018. Resilience of regional transportation networks subjected to hazard-induced bridge damages. Journal of Transportation Engineering, Part A: Systems 144(10): Article 04018062.

Utasse, M., V. Jomelli, D. Grancher, F. Leone, D. Brunstein, and C. Virmoux. 2016. Territorial accessibility and decision-making structure related to debris flow impacts on roads in the French Alps. International Journal of Disaster Risk Science 7(2): 186-197.

Vugrin, E.D., M.A. Turnquist, and N.J.K. Brown. 2014. Optimal recovery sequencing for enhanced resilience and service restoration in transportation networks. International Journal of Critical Infrastructures 10(3-4): 218-246.

Zhou, Y., J. Wang, and H. Yang. 2019. Resilience of transportation systems: Concepts and comprehensive review. IEEE Transactions on Intelligent Transportation Systems 20(12): 4262-4276. 